\title{
Management and Outcome of Pregnant Women with HIV Acquired by Vertical Transmission
}

\author{
Verónica Serrano de la Cruz Delgado*, Alicia Martínez Varea, María José Núñez Valero, \\ Vicente Diago Almela, Vicente Maiques Montesinos, Amparo García Tejedor, \\ Alfredo Perales Marín
}

\author{
HUP La Fe, Valencia, Spain \\ Email: 'Vero SDLCD@msn.com, martinez.alicia.v@gmail.com, claris mi@hotmail.com, \\ diagovicalm@gva.es, maiquesmontesinos@gmail.com, agarciat@bellvitgehospital.cat, \\ perales_alf@gva.es
}

Received 22 July 2015; accepted 22 August 2015; published 25 August 2015

Copyright (C) 2015 by authors and Scientific Research Publishing Inc.

This work is licensed under the Creative Commons Attribution International License (CC BY).

http://creativecommons.org/licenses/by/4.0/

(c) (i) Open Access

\begin{abstract}
The majority of children with perinatally acquired human immunodeficiency virus (HIV) do not survive beyond childhood but this is changing due to the benefit of antiretroviral therapy for the perinatally infected cohort, so affected children are now approaching the age to be mothers. The aim of this article is to evaluate the outcomes of pregnant women with HIV acquired by vertical transmission in our centre and encourage the results obtained in "Thirty Years Later: Pregnancies in Female Perinatally Infected with Human Immunodeficiency Virus-1", a review article published in this journal in 2012 [2]. We report 6 patients with eight pregnancies with HIV acquired by vertical transmission. They delivered a total of nine newborns between 2004 and 2013. In any case, mother-to-child transmission was reported. The management of pregnant women with HIV acquired by vertical transmission is complicated, since the patients are the most of the cases young and the compliance to the treatment may be poor. The prematurity was the more frequent complication and the cesarean section was the more frequent form of ending. In our case series, mother-to-child transmission was absent.
\end{abstract}

\section{Keywords}

HIV, Perinatally Acquired HIV, Vertical Transmission HIV, Pregnancy

\footnotetext{
"Corresponding author.
}

How to cite this paper: de la Cruz Delgado, V.S., Varea, A.M., Valero, M.J.N., Almela, V.D., Montesinos, V.M., Tejedor, A.G. and Marín, A.P. (2015) Management and Outcome of Pregnant Women with HIV Acquired by Vertical Transmission. Open Journal of Obstetrics and Gynecology, 5, 470-474. http://dx.doi.org/10.4236/ojog.2015.59068 


\section{Introduction}

The first pregnancy in a patient with perinatally acquired AIDS was documented during the early 1980s. Previously, the infection usually progressed rapidly to death and the majority of perinatally acquired HIV children did not survive beyond reproductive age. A significant decrease in pediatric AIDS deaths has been produced due to the benefit from antiretroviral therapy for the perinatally infected cohort [1]. HIV treatment has led to major clinical advances in HIV care and has transformed HIV/AIDS from a uniformly fatal disease to a chronic disease. Combined HAART for perinatally HIV acquired children has prolonged their survival, and in the past two decades many have been able to achieve the adulthood. So, perinatally infected children are getting adolescence and young adulthood in a better health status, which lets them be mothers.

The aim of this article is to evaluate the outcome of pregnant women with HIV acquired by vertical transmission in our centre and encourage the results obtained in "Thirty Years Later: Pregnancies in Female Perinatally Infected with Human Immundodeficiency Virus-1”, a review article published in this journal in 2012 [2].

\section{Patients and Methods}

It has been carried out a retrospective study about all pregnant women with vertically-acquired HIV whose pregnancies were followed in our tertiary Hospital between 2004 and 2013. A total of six patients with HIV acquired by vertical transmission have been analyzed. They delivered eight pregnancies and nine newborns between 2004 and 2013.

A female is considered to have perinatally acquired HIV infection if her mother was infected by HIV during pregnancy, labor or delivery according to clinical records, or if she is found to be infected by HIV during infancy or early childhood without another explanation for exposure [2].

We evaluate HIV treatment, compliance, gestational age at booking in the maternity, gestational age at delivery, preterm birth being defined by gestational age below 37 weeks, CD4 cell counts and HIV-1 RNA VLs obtained early and close to the time of delivery. A child was considered infected if HIV-1 DNA or RNA polymerase chain reaction was positive on 2 samples or HIV-1 antibodies were detected at 6 months of age, or uninfected in other cases.

\section{Results}

All patients took HAART during pregnancy, at least at the end of it. All pregnant received AZT during delivery (loading dose $2 \mathrm{mg} / \mathrm{kg}$ in half an hour, and maintenance dose $1 \mathrm{mg} / \mathrm{kg} /$ hour until delivery). All the newborns received zidovudine. Breastfeeding was artificial in all cases.

In Table 1, there are exposed all the studied cases.

The average age of women was 20 years (range 16 - 26). Six women underwent her first pregnancy (87.5\%). Seven pregnancies were single and one was twin. The twin pregnancy was dichorionic with small for gestational age fetuses, although after birth their weigh percentiles were above 10. One pregnant woman suffered pneumonia, but got over without incidents, and in another one was diagnosed a high-grade squamous intraepithelial lesion (HSIL) which is now still being controlled in our Hospital. The remainder pregnant women underwent uncomplicated pregnancies. At 36 weeks of gestation, 4 pregnants (50\%) had viral load below 1000 copies $/ \mathrm{ml}$. The other pregnant had a viral load above 1000 copies $/ \mathrm{ml}$. In the last four cases the compliance of the treatment was very poor. All pregnancies were delivered by a cesarean section, only four of them was urgent, one due to the patient was in labor and the remaining three because they suffer premature rupture of membranes. In some cases, the vaginal delivery was possible but the patients who were suitable for a vaginal delivery refused it because they were afraid about transmitting their disease to their children. The average gestational age at term was 36 weeks (33 - 38), only 2 pregnancies finished before 37 weeks of pregnancy (25\%). The average weight of the newborns was $2518 \mathrm{~g}$ (1820 - $3325 \mathrm{~g}$ ), no one was under percentile 10 (average p 31\%, range 15 - 85), so no one had low weigh. In any case mother-to-child transmission was reported.

\section{Discussion}

The first case report of pregnancy (1998), the patient was 14 years and delivered an HIV negative infant a term [3]. Badell et al. published a review in 2012 about pregnancies in females perinatally infected with human immunodeficiency virus-1, and we want to report our experience with 8 patients who have been treated in the last 


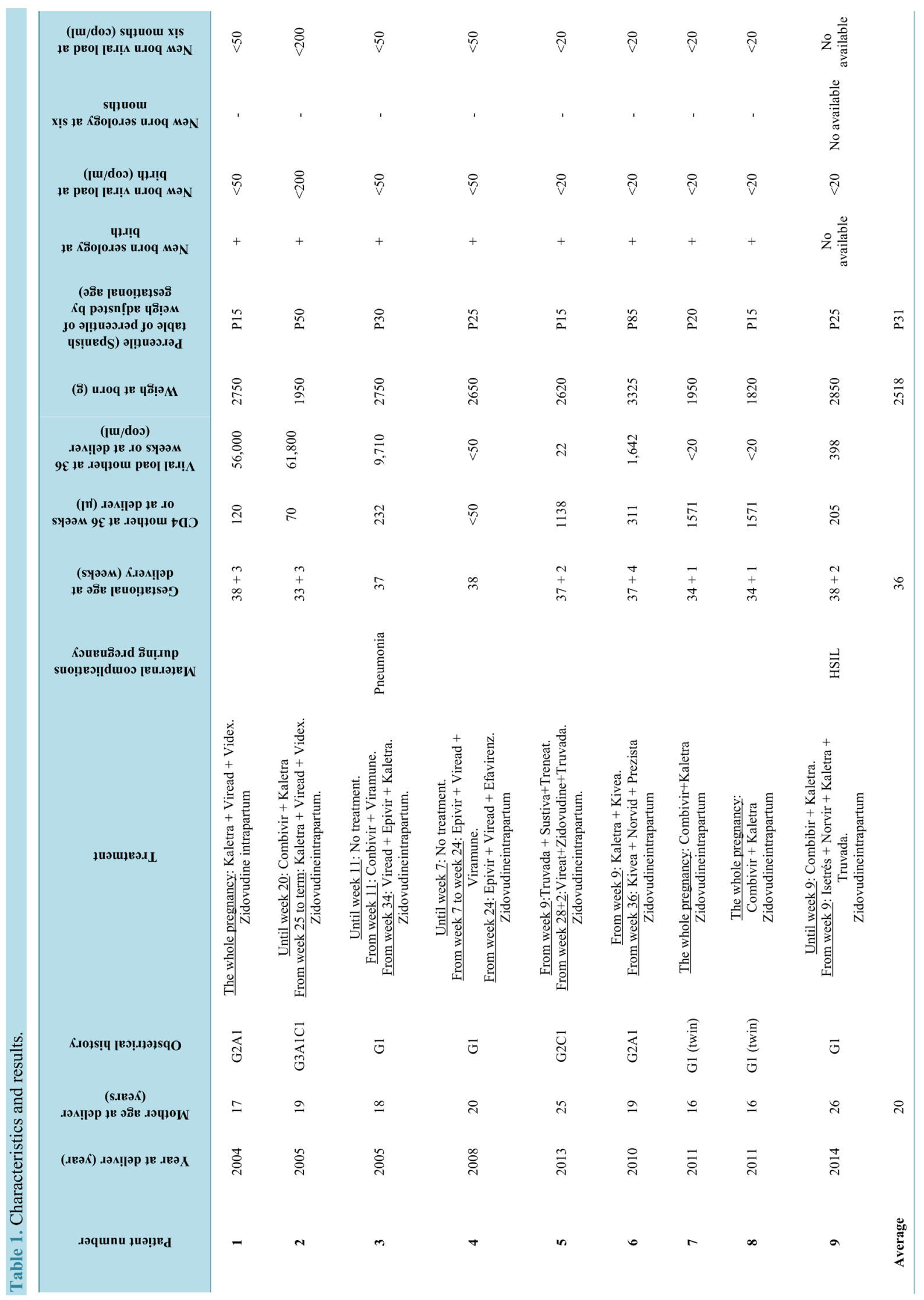


years in our center. In the cited review, the sum of reviewed cases reaches 132 pregnancies, although not every of them arrived a term, in some reports the rate of abortion (spontaneous or volunteers) arrives to the $48 \%$.

Analyzing the results, as in the other studies published, in our population VIH mothers are very young, the average age of women was 20 years old (range 16 - 26). This fact adds another risk for the pregnancies in this kind of population, and contributes to lower compliance with measures of fetal and maternal care necessary for the proper development of pregnancy. In our centre the average gestational age at term was 36 weeks. The average weight of the newborns was $2518 \mathrm{~g}$, being the neonatal percentile average $31 \%$; no one was low weigh. In a study by William et al., they found that the median age of the first pregnancy was 18.5 years and the mean gestational age at the time of delivery was 38 weeks, something which has surprised us considerably because in the most of case reports the mean age is premature to term [4]. Other authors found that preterm delivery occurs up in $44 \%$ of cases [5] [6]. In our experience, the prematurity rates is lower, reached $25 \%$, because two of eight pregnancies finished before 37 weeks, so related to prematurity we agree what Martina L. Badell claimed in her review, VIH pregnancies and prematurity are connected. At the moment, the etiology of the increased risk for prematurity in this population is unknow. Most studies propose the number of preterm is higher among HIV infected women and they suggest that is due to the young age of the mothers, which is a risk factor of preterm birth, and the treatment, as HAART has also been associated with preterm delivery [2] [7]. In our database, we can see that the patient with the highest viral load, delivered earlier than the other women, exactly at 33 weeks. Maybe, although HAART and mother age play a paper in prematurity, viral load can also be related to. Furthermore than the morbidities prematurity can produce, it is important for these infants' health owing to it has relevance on the selection of HAART when they born, because of in these new borns it can only be used zidovudine due to the medication dosing is only available in that drug. In addition, as they are premature, the risk of renal or hepatic complications due to treatment is higher. Moreover, premature newborns have usually an increased risk for viral resistance secondary to a short duration and amount of HAART exposure in the mother [2].

The treatment compliance is low in this type of patients because the treatments are long and hard, and the patients are teenager or young; consequently the viral load is usually high [8]. In our experience, a correct compliance was achieved by $55.5 \%$ of the patients who got lower viral loads at the moment of the delivery. Our experience is too small to draw conclusions, but the patient whose compliance was the worst, reached higher viral load and delivered more prematurely. There is no specific data about this issue in the publications we have review, and it would be interesting because is a weak point which obstetricians could work to get better maternal and perinatal results because it is well known that HAART therapy reduces the influence of the perinatal risk factors on vertical HIV transmission [9]. In any case, detection and track of these patients is important in order to start pregnancy care from the beginning and get better results.

Women who acquired VIH by mother-to-child transmission have an increased risk of delivery by cesarean section. The recommendations in the United States point that the delivery by cesarean section is safer if the pregnant has viral load above 1000 copies/ml [2]. However, although some of our patients were suitable for a vaginal delivery because they viral load was lower than this figure or even undetectable, they refused it because they were afraid of transmitting their illness to their children. All the new borns were health, any of them were infected. Nevertheless, as Badell et al. suggest, we should educate our patients because they are not completely aware about the complications of a cesarean section in the moment of the delivery as well as in future pregnancies, especially repeated cesarean. So in cases with viral load under 1000 copies/ml when vaginal delivery is safe, we should encourage women for parturition as the guidelines recommend. Fortunately, post-cesarean complications are not significantly upper in HIV-infected women treated with HAART compared to non-HIV-infected women. Prematurity and low CD4+ cell count have been described as risk factors for major complication. Thus, cesarean delivery is as safe in these patients as in healthy women [10]. In summary, we need encourage women and experience vaginal delivery in these patients to assess advantages and disadvantages of this way in this population, although in people with HIV acquired in adult life, we know vaginal delivery is safe if the viral load is under 1000 copies $/ \mathrm{ml}$.

Anyway, young infected women need additional counseling on sexuality, reproductive health and contraception; and if they get pregnant we should insist on proper HAART management, as well as social support to achieve a maximal viral suppression, which is essential to prevent mother-to-child transmission. This is a huge task, and it should involve all levels of care to achieve targets.

In conclusion, the management of pregnant women with HIV acquired by vertical transmission is complicated, since in most cases the women are young and the compliance to the treatment may be poor. Combined HAART 
during pregnancy should be a strict treatment in order to achieve the maximal viral suppression at the moment of the delivery. Even when the viral load is controlled, these pregnancies suffer complications like prematurity. In our case series, mother-to-child transmission was absent. Cesarean section was performed in all of our patients because those who were suitable for a vaginal delivery refused it.

\section{Conflict of Interests}

The authors declare that there is no conflict of interests regarding the publication of this paper.

\section{Acknowledgements}

We thank all obstetrical staff in HUP LA FE in Valencia (Spain) for their help.

\section{References}

[1] WHO (2014) Data on the Size of the HIV/AIDS Epidemic: Data by WHO Region. World Health Organization, Geneva. http://apps.who.int/gho/data/node.main.619?lang=en

[2] Badell, M.L. and Lindsay, M. (2012) Thirty Years Later: Pregnancies in Females Perinatally Infected with Human Immunodeficiency Virus-1. AIDS Research and Treatment, 2012, Article ID: 418630, 6 p. http://dx.doi.org/10.1155/2012/418630

[3] Crane, S., Sullivan, M., Feingold, M. and Kaufman, G.E. (1998) Successful Pregnancy in an Adolescent with Perinatally Acquired Human Immunodeficiency Virus. Obstetrics \& Gynecology, 92, 711. http://dx.doi.org/10.1016/S0029-7844(98)00292-0

[4] Williams, S.F., Keane-Tarchichi, M.H., Bettica, L., Dieudonne, A. and Bardeguez, A.D. (2008) Pregnancy Outcomes in Young Women with Perinatally Acquired Human Immunodeficiency Virus-1. American Journal of Obstetrics and Gynecology, 200, 149e1-149e5.

[5] Thorne, C., Townsend, C.L., Peckham, C.S., Newell, M.L. and Tookey, P.A. (2007) Pregnancies in Young Women with Vertically Acquired HIV Infection in Europe. AIDS, 21, 2552-2556. http://dx.doi.org/10.1097/QAD.0b013e3282f08b5f

[6] Beckerman, K., Giovanniello, A. and Wright, R. (2011) Retrospective Cohort Comparing Pregnancy Outcome among Perinatally Infected Women Compared to Sexually Infected. Proceedings of the IDSA Annual Meeting, Boston, 20-23 October 2011, 20-23.

[7] Townsend, C.L., Schulte, J., Thorne, C., et al. (2010) Antiretroviral Therapy and Preterm Delivery-A Pooled Analysis of Data from the United States and Europe. BJOG, 117, 1399-1410. http://dx.doi.org/10.1111/j.1471-0528.2010.02689.x

[8] Rudy, B.J., Murphy, D.A., Harris, D.R., Muenz, L. and Ellen, J. (2010) Prevalence and Interactions of Patient-Related Risks for Non-Adherence to Antiretroviral Therapy among Perinatally Infected Youth in the United States. AIDS Patient Care and STDs, 24, 97-104. http://dx.doi.org/10.1089/apc.2009.0198

[9] Garcia-Tejedor, A., Maiques, V., Perales, A. and Lopez-Aldeguer, J. (2009) Influence of Highly Active Antiretroviral Treatment (HAART) on Risk Factors for Vertical HIV Transmission. Acta Obstetricia et Gynecologica Scandinavica, 88, 882-887. http://dx.doi.org/10.1080/00016340903062836

[10] Maiques, V., Garcia-Tejedor, A., Diago, V., Molina, J.M., Borras, D., et al. (2010) Perioperative Cesarean Delivery Morbidity among HIV-Infected Women under Highly Active Antiretroviral Treatment: A Case-Control Study. European Journal of Obstetrics \& Gynecology and Reproductive Biology, 153, 27-31. http://dx.doi.org/10.1016/j.ejogrb.2010.07.001 